\title{
ON THE NEAR-WAKE OF A GROUND-EFFECT DIFFUSER WITH PASSIVE FLOW CONTROL
}

\author{
Obinna Ehirim $^{1)}$, Kevin Knowles ${ }^{1)}$, Alistair Saddington ${ }^{1)^{*}}$, Mark Finnis ${ }^{1)}$ and Nicholas Lawson ${ }^{2)}$ \\ ${ }^{1)}$ Aeromechanical Systems Group, Centre for Defence Engineering, Cranfield University, Shrivenham, Swindon, \\ SN6 8LA, UK \\ ${ }^{2}$ National Flying Laboratory Centre, Cranfield University, Cranfield, Bedford, MK43 0AL, UK
}

(Received date $\quad$; Revised date $\quad$; Accepted date $\quad$ ) * Please leave blank

\begin{abstract}
A ground-effect diffuser is an upwardly-inclined section of an automobile's underbody which increases aerodynamic performance by generating downforce. To understand the diffuser flow physics (force behaviour, surface and off-surface flow features), we established the near-wake (within one vehicle width of the base) velocity profiles and flow structures of an automotive ground-effect diffuser using a bluff body with a 17 degree slanted section forming the plane diffuser ramp surface (baseline geometry), and endplates extending along both sides of the ramp. Wind tunnel experiments were conducted at a Reynolds number of 1.8 million based on the bluff body length, and laser Doppler velocimetry was used to measure two-dimensional velocity components on three planes of the diffuser near-wake. We also measured the velocity field in the near-wake of diffusers with modified geometry (with an inverted wing or a convex bump) as passive flow control devices. The near-wake velocity profiles indicated that the passive flow control methods increased the diffuser flow velocity and that the longitudinal vortices along the diffuser determined the shape of the flow structures in the near-wake of the diffuser bluff body.
\end{abstract}

KEY WORDS : Laser Doppler Velocimtery, Ground Effect, Diffuser, Flow Control

\section{NOMENCLATURE}

As: $\quad$ bluff body frontal area, $\mathrm{m}^{2}$

$C_{D}: \quad$ drag coefficient, $D /\left(q_{\propto} A s\right)$

$C_{L}: \quad$ lift coefficient, $L /\left(q_{\propto} A S\right)$

$d: \quad$ diffuser half width, $\mathrm{m}$

$D: \quad$ aerodynamic drag, $\mathrm{N}$

$D_{p}: \quad$ seeding particle diameter, $\mathrm{m}$

$f: \quad$ focal length of the LDV probe lens, $m$

$h$ : $\quad$ bluff body model ride height, $\mathrm{m}$

$l$ : $\quad$ bluff body length, $\mathrm{m}$

$L \quad$ aerodynamic lift (positive upwards), $\mathrm{N}$

LDV: laser Doppler velocimetry

$q_{\infty}: \quad$ freestream dynamic pressure $\left(\rho U_{\infty}{ }^{2} / 2\right), \mathrm{Pa}$

$R e: \quad$ Reynolds number based on $l$ and $U_{\infty}$

$T_{c} \quad$ flow characteristic timescale, $\mathrm{s}$

$U_{\infty}: \quad$ freestream velocity, $\mathrm{ms}^{-1}$

$U_{w}: \quad$ downstream flow velocity, $\mathrm{ms}^{-1}$
$U: \quad$ total velocity in $x-y$ plane $\sqrt{u^{2}+v^{2}}, \mathrm{~ms}^{-1}$ $u^{\prime}$ : root mean square of turbulent velocity fluctuations, $\mathrm{ms}^{-1}$

$\Delta U: \quad$ plane diffuser $U$ - modified diffuser $U, \mathrm{~ms}^{-1}$

$\Delta u^{\prime}: \quad$ plane diffuser $u^{\prime}$ - modified diffuser $u^{\prime}, \mathrm{ms}^{-1}$

$u: \quad$ velocity component in $x$ direction, $\mathrm{ms}^{-1}$

v: $\quad$ velocity component in $y$ direction, $\mathrm{ms}^{-1}$

$x$ : $\quad$ Cartesian coordinate: $x$ is positive downstream of origin at the start of underbody flat section (Fig 4a)

$y$ : $\quad$ Cartesian coordinate: $y$ is positive upwards of origin on the ground plane (Fig 4a)

$z$ : Cartesian coordinate: $z$ is positive portside of origin on the centreline of the body (Fig 4a)

$\Gamma$ : $\quad$ circulation, $\mathrm{m}^{2} \mathrm{~s}^{-1}$

$\mu: \quad$ dynamic viscosity $\mathrm{kgm}^{-1} \mathrm{~s}^{-1}$

$\rho: \quad$ air density, $\mathrm{kgm}^{-3}$

$\rho_{p}: \quad$ seeding particle density, $\mathrm{kgm}^{-3}$

\section{INTRODUCTION}

A ground-effect diffuser is a ramped surface on the aft underbody of a racing car that makes a considerable

\footnotetext{
* Corresponding author:

a.j.saddington@cranfield.ac.uk
} 
impact on aerodynamic performance by contributing the most downforce (Toet, 2013). The diffuser can be described as an increasing area duct when in close proximity to the road surface, and its aerodynamic performance is sensitive to the vehicle's ride height (Zhang et al., 2006). When positioned close to the road surface, the airflow between the car underbody and the road surface is constrained as the racing car travels forwards. The constrained airflow accelerates through the diffuser inlet as a high-velocity flow with low static pressure, and exits the diffuser as a low-velocity flow with high static pressure (Sovran, 1994). Downforce is generated by the low-pressure suction generated at the diffuser inlet (Hucho, 1998) and this increases the grip between the tyres and the road surface, allowing faster cornering (Katz, 2006).

Several wind tunnel investigations have been carried out using a bluff body with an underbody diffuser to address the three-dimensional (3-d) flow physics of ground-effect diffusers. George (1981) tested a diffuserequipped bluff body with and without end plates, and with ramp angles of $10^{\circ}$ and $20^{\circ}$. In addition, the diffuser bluff body was positioned at pitch angles ranging from $10^{\circ}$ (nose up) to $+35^{\circ}$ (nose down) (defined at the centrepoint of the bluff body's sides). When the pitch angle was increased (starting from zero) by $5^{\circ}$ increments for the $20^{\circ}$ diffuser body, a longitudinal vortex pair formed towards the rear of the underbody at low angles of attack and as the pitch angle further increased, the vortices moved forward and strengthened. The flow behaviour was attributed to the induced inflow which prevented the formation of a separation bubble on the diffuser ramp surface. Even at the extreme pitch angle of $+35^{\circ}$, where a separation bubble formed upstream on the underbody, the strong vortices caused the separated flow to reattach downstream on the underbody. However, at a pitch angle of $-10^{\circ}$ with a $10^{\circ}$ diffuser ramp, the longitudinal vortex flow was established by the upstream separating free shear layer.

George and Donis (1983) investigated the aerodynamic effect of diffuser endplates and discovered that when the endplates for $10^{\circ}$ and $15^{\circ}$ diffusers are sealed to the ground, the diffuser inflow induced by the longitudinal vortex pair is weaker and there is less downforce, but the opposite effect is observed when the diffuser endplates are unsealed.

Cooper et al. (1998, 2000) investigated the relationship between diffuser length and area ratio under fixed ground and moving ground conditions using a bluff body with two diffuser lengths $(25 \%$ and $75 \%$ of bluff body length). When the boundary layer of the diffuser was considered, they found that the diffuser effective area ratio for a given geometric area is larger under moving ground than fixed ground conditions. For a particular diffuser length, a given diffuser pressure recovery can therefore be achieved with a smaller geometric area ratio under moving ground conditions. Although fixed ground wind tunnel conditions are not representative of a racing car traveling along a track, longitudinal vortex flow structures are generated within the diffuser due to the inflow caused by the pressure differential.

Two-dimensional (2-d) velocity vector plots taken at a spanwise plane near the diffuser exit under fixed ground conditions by particle image velocimetry (PIV) revealed the existence of vortical structures near the endplates of a $13^{\circ}$ bluff body diffuser (Jowsey and Passmore, 2010; Jowsey, 2013). Comparative velocity profiles from computational investigations using the large-eddy simulation (LES) turbulent flow modelling technique agreed with these wind tunnel investigations (Puglisevich and Page, 2011; Puglisevich, 2013). However, the longitudinal vortices from the wind tunnel experiments occupied a larger area of the diffuser crosssection than the vortical structures captured by computational simulations. The disparity provides an explanation for the lower diffuser suction levels predicted by computational fluid dynamics compared to wind tunnel experiments.

Force measurements (Figure 1) on a diffuserequipped bluff body under moving ground wind tunnel conditions have revealed the presence of four distinct diffuser flow regimes (Senior and Zhang, 2001; Senior, 2002). In the force-enhancement flow regime (region $A$ ), downforce $\left(C_{L}\right)$ increased as the bluff body is gradually lowered from an elevated ride height. A further increase in downforce is observed in region $\mathrm{B}$ with a further decrease in ride height until maximum downforce is achieved at a critical ride height $(h / d=0.217)$. When the ride height is reduced further, downforce reduction occurred in the regions $\mathrm{C}$ and $\mathrm{D}$ flow regimes.

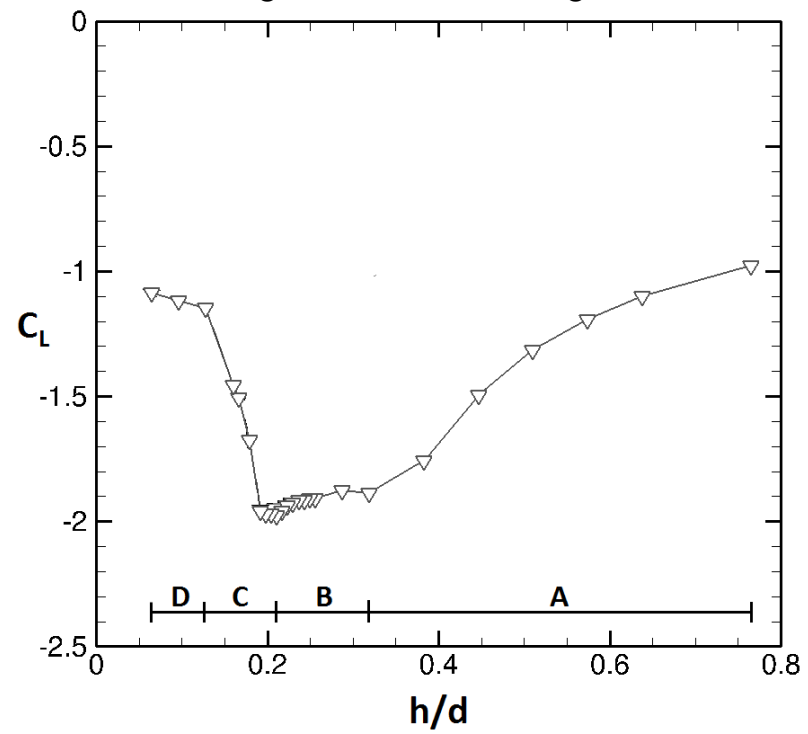

Figure 1. Downforce measurements and flow regimes (A to D) over the ride height interval $h / d=0.764$ to 0.064 (Senior and Zhang, 2001; Senior, 2002). 
Laser Doppler anemometry velocity measurements taken by Zhang et al., 2004 on a cross-plane near the diffuser exit provided insight into the diffuser flow physics with an indication that the suction-enhancing longitudinal vortex pair development along the lengthwise sides of the diffuser ramp is responsible for the downforce characteristics. In the force-enhancement flow regime, a concentrated counter-rotating pair of vortices with a high-speed axial core was prevalent close to the end plates. In the maximum-downforce flow regime, the size of the vortical structures increased due to the greater vortex strength in the early part of the diffuser, but vortex diffusion induced a low axial speed core towards the diffuser exit. However, an asymmetric flow was observed in the force-reduction regime, with the disappearance of one of the vortex pair (vortex breakdown). The surviving edge vortex was weak, and flow reversal due to extensive flow separation occurred in much of the area previously occupied by the disrupted vortex. In the low-downforce flow regime, the surviving edge vortex was weakened further with an increasingly dominant boundary-layer flow entering the diffuser, and flow recirculation dominating the diffuser flow. Continuing to reduce ride height further leads ultimately to complete separation of the flow over the entire width of the inlet of the diffuser and the consequential loss of both vortices. During this vortex transition regime, LDA data from points in the wake has shown evidence of a number of dominant frequencies. These frequencies are indicative of highly unsteady vortical flow structures, expected prior to the breakdown of these wake structures. This transition regime will be the subject of a future paper.

The aerodynamic downforce generated by a diffuser can be enhanced by inducing a secondary pressure drop region towards the diffuser exit via a local reduction in the cross-sectional area (Ehirim, 2017). As established by Bernoulli's principle, the lower pressure results in greater velocity. Thus the modification of the area close to the diffuser exit to include, for example, a bump causes an increase in velocity with a corresponding pressure drop at the location and, as a result, local downforce increases. Such passive flow control methods achieved by geometric alterations in the diffuser further facilitate the suction effect generated by the diffuser.

Here we used laser Doppler velocimetry (LDV) to capture the near-wake velocity profiles of a diffuser bluff body similar to that used in the investigation reported by Senior, 2002. For the purposes of this article the nearwake is considered to be within one vehicle width of the base. The objective of this study was to use the diffuser velocity profiles to confirm the previously-described flow physics (force behaviour, surface and off-surface flow features) and understand the mechanisms in more detail. We also investigated the near-wake velocity profiles of the diffuser with passive flow control methods that enhance downforce by increasing flow velocity and lowering the static pressure distribution downstream of the diffuser inlet (Ehirim, 2017).

\section{DESCRIPTION OF EXPERIMENTS}

\subsection{Wind Tunnel Facility}

All tests were conducted in the Cranfield University DS Houghton wind tunnel at Shrivenham, a $2.74 \mathrm{~m}$ by $1.66 \mathrm{~m}$ closed-return, three-quarter open-jet tunnel (Figure 2). The facility includes a continuous-belt rolling road, which represents moving ground conditions and is synchronised with the freestream velocity of the tunnel. Also included is a boundary layer removal system near the nozzle exit of the tunnel. The distribution and a priori optimisation of suction ensures a minimal boundary layer growth on the belt (local displacement thickness of $<0.04$ $\mathrm{mm}$ ). Lifting of the belt due to aerodynamic loading is prevented by the equal distribution of suction underneath the rolling road. The freestream turbulence intensity is $\sim 0.3 \%$. Chiller units were employed to control the air and rolling-road temperatures. The wind tunnel facility is described in detail by Knowles and Finnis, 1998.

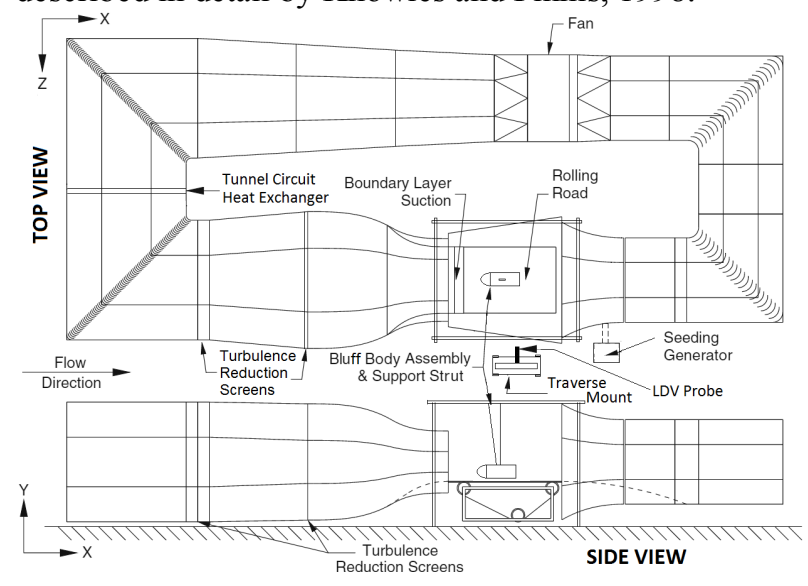

Figure 2. Schematic representation of the DS Houghton wind tunnel facility and experimental setup.

\subsection{Bluff-Body Test Models}

The main component of the experimental model was a bluff body with a $17^{\circ}$ slanted plane surface used as the diffuser ramp, and with $5 \mathrm{~mm}$-thick endplates extended along the edges of the diffuser (Figure 3). The bluff-body model was constructed from aluminium with a SikaBlock polyurethane nose section, and the dimensions (0.326 m height, $0.314 \mathrm{~m}$ width, $1.315 \mathrm{~m}$ length) were similar to those reported by Senior (2002). The bluff body was mounted on the wind tunnel strut above the rolling-road. The diffuser passive flow control components comprised a convex bump (0.0056 $\mathrm{m}$ height, $0.304 \mathrm{~m}$ width, $0.092 \mathrm{~m}$ length) on the diffuser ramp positioned near the diffuser exit, or a modified NASA 
GA(W) type LS(1)-0413 inverted wing (Zerihan and Zhang, 2000) with a chord length of $0.0837 \mathrm{~m}$ and a span between the end plates of $0.304 \mathrm{~m}$.

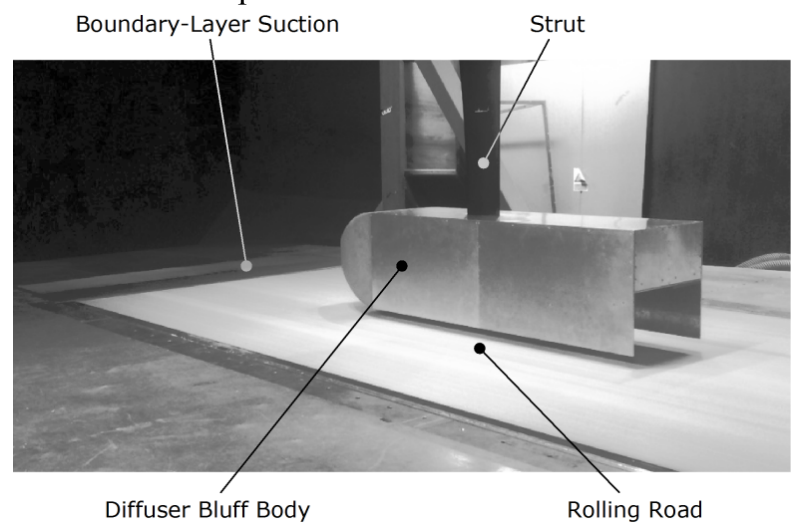

Figure 3. Bluff body test model in the $2.74 \mathrm{~m}$ by $1.66 \mathrm{~m}$ DS Houghton wind tunnel.

The inverted wing was mounted close to the exit of the diffuser flow channel at a distance of $0.01435 \mathrm{~m}$ from the diffuser ramp surface (about 50\% of the estimated turbulent boundary layer thickness). Cross-sections of the bluff body model with and without the passive flow control components are shown in Figures 4 and 5.

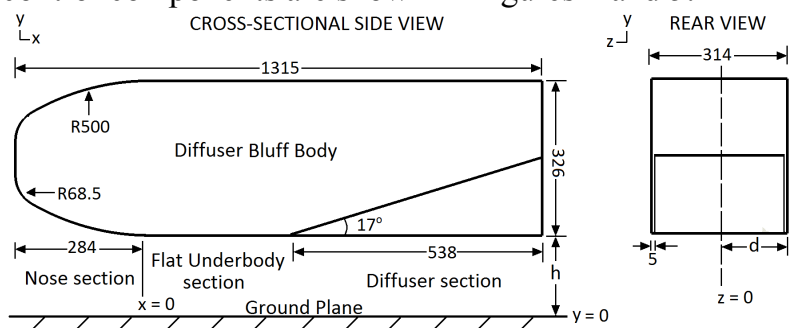

Figure 4. Cross-sectional and rear views of the baseline (plane diffuser) bluff body with no passive flow control components (dimensions in $\mathrm{mm}$ ).

\subsection{Laser Doppler Velocimetry System}

LDV is a laser-based system used for the non-intrusive, pointwise measurement of mean and instantaneous velocity in a fluid flow (McKeon et al., 2007). We used a TSI LA6000 argon-ion LDV flow-field measurement system and a probe with a lens operating at a focal length of $f=2.29 \mathrm{~m}$ with interrogation volume dimensions of $0.55 \mathrm{~mm}$ by $0.55 \mathrm{~mm}$ by $5.01 \mathrm{~mm}$. The LDV system (Figure 6) included a two-component TR160 series 83 $\mathrm{mm}$ fibre-optic probe (with an XPD60 series beam expander), two $1 \mathrm{~W}$ multicolour beam generators (separator) with two Bragg cells producing beams with wavelengths of 514 and $488 \mathrm{~nm}$, four fibre couplers (two for each beam generator), a photodetector module, and a signal processor. The probe was mounted on a bidirectional automated traverse system and was used to measure velocity components $(u, v)$ in a 2 -d plane $(x-y)$ with data acquired in coincident mode.

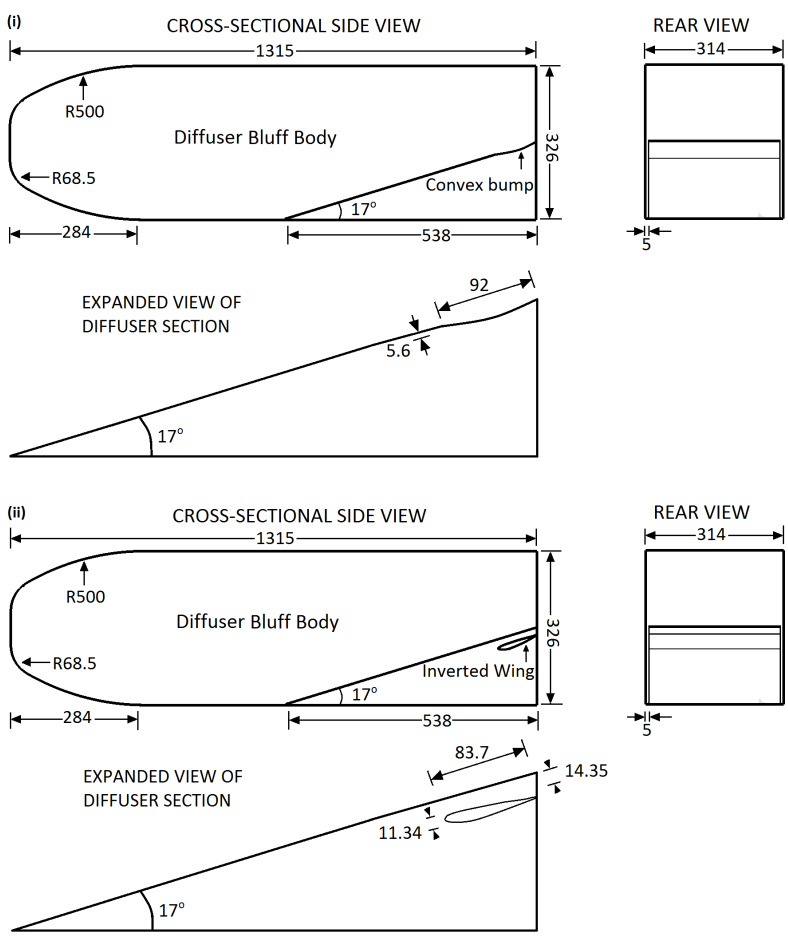

Figure 5. Cross-sectional and rear views of the modified diffuser bluff body with passive flow control components: (i) with the convex bump, and (ii) with the inverted wing (dimensions in $\mathrm{mm}$ ).

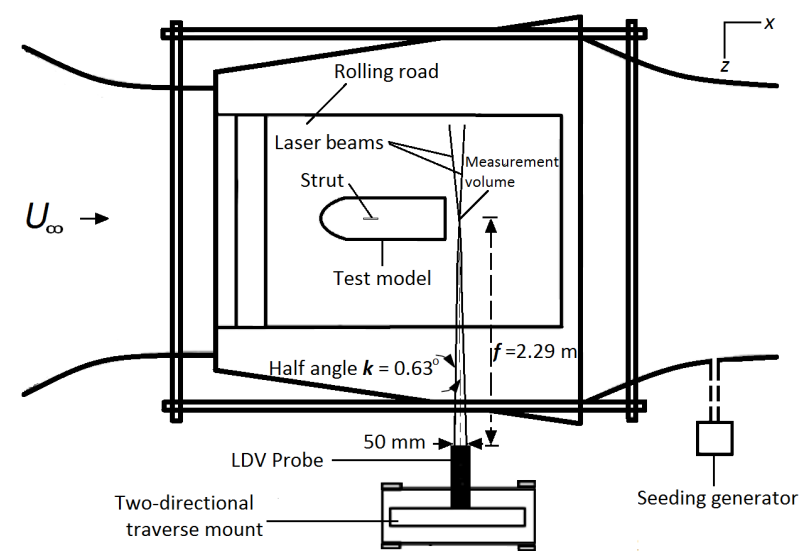

Figure 6. A schematic with dimensions of the LDV system setup in the wind tunnel test section.

The velocity limits for the LDV channels that captured the $u, v$ velocity components were; $-31.73 \mathrm{~ms}^{-1}$ to $49.87 \mathrm{~ms}^{-1}$ and $-30.10 \mathrm{~ms}^{-1}$ to $47.30 \mathrm{~ms}^{-1}$, respectively. Signals were processed using a burst spectrum analyser controlled by TSI Flow Sizer 64 v4.2.0 software. Flow seeding particles of $0.9 \mu \mathrm{m}$ mean diameter were generated from a solution of di (2- 
ethylhexyl) sebacate (DEHS) with a PIVTECH Part45 aerosol seeding generator.

\subsection{Experimental procedure}

The experiments were carried out at a freestream and rolling-road velocity of $20 \mathrm{~ms}^{-1}$, representing a Reynolds number of 1.8 million based on the bluff-body length. The model has a wind tunnel blockage ratio of $2.24 \%$ but the use of a wind tunnel with an open test section negated the need for blockage corrections. Velocity measurements were taken at three spanwise planes (Figure 7) located at near-wake streamwise locations $(z / d=0.49 ; 0$; -0.49) for non-dimensional model ride heights of $h / d=$ 0.191 and 0.153 corresponding to $0.03 \mathrm{~m}$ (maximum force flow regime) and $0.024 \mathrm{~m}$ (force reduction flow regime). Three-hundred and eighty data points were positioned on an $x$-y planar grid with dimensions of $323 \mathrm{~mm}$ by $306 \mathrm{~mm}$ in the direction of the freestream. Measurements began $15 \mathrm{~mm}(x / d=6.67)$ downstream of the model and were recorded at a spatial resolution of $17 \mathrm{~mm}$ in both the $x$ and $y$ directions. We took 2000 samples at each measurement point over a sampling time of $20 \mathrm{~s}$ and the ensemble average of velocity data was extracted. A settling time of $5 \mathrm{~s}$ was included after each probe movement to allow for the decay of any probe vibration.

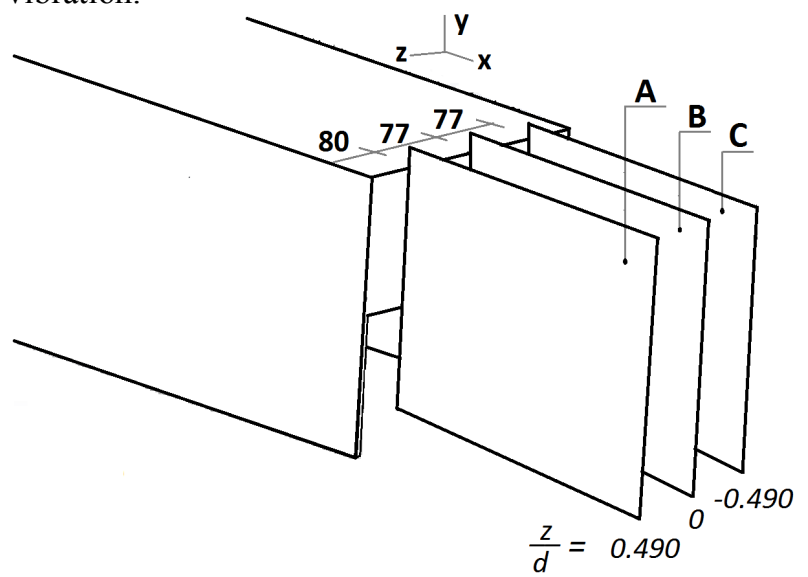

Figure 7. Measurement planes (A, B, C) of the diffuser near-wake (dimensions in $\mathrm{mm}$ ).

\subsection{Error Analysis}

Experimental errors and uncertainties were calculated as described by Knowles, 2005 who carried out experiments in the same wind tunnel. The model ride height was measured to an accuracy of $\pm 0.02 \mathrm{~mm}$ using a drop height gauge and the yaw angle was set to within $\pm 0.05^{\circ}$. The freestream velocity and rolling-road speed were regulated by the wind tunnel control system to within $\pm 0.06 \mathrm{~ms}^{-1}$ and $\pm 0.02 \mathrm{~ms}^{-1}$ respectively. The LDV probe can measure velocities at any point within the measurement volume. This implied a systematic error in the measurement location within the dimensions of the
LDV measurement volume. The maximum location error where the particle velocity is measured was assumed to be $50 \%$ of the $x-y-z$ dimensions of the measurement volume. This equates to systematic errors of $\pm 0.28 \mathrm{~mm}$, $\pm 0.28 \mathrm{~mm}$ and $\pm 2.5 \mathrm{~mm}$ in the $x, y$ and $z$ reference axes, respectively.

The seeding response error due to the inability of the seeding particles to follow the flow was calculated as described by Dring, 1982. The methodology associates the acceleration of a particle in a surrounding fluid to its Stokes number, and if the Stokes number is $<0.01$ then the maximum velocity error is the same as its Stokes number. The Stokes number, defined as the ratio of the particle response time to the characteristic time-scale of the flow, $\rho_{p} D_{p}^{2} /\left(18 \mu T_{c}\right)$, was estimated to be $\sim 2.8 \times 10^{-4}$, indicating that the seeding response error was insignificant.

In evaluating the mean velocity, the velocity bias was eliminated by applying the particle transit time $t$ as the sample weighting factor, which is inversely proportional to the sample velocity (Buchave et al., 1979). This was necessary because at a particular point in a uniformly-seeded flow, velocity fluctuations greater than the mean value can capture more samples than a comparable velocity fluctuation below the mean value. As a result, the calculated mean velocity will tend to be biased towards velocities above the mean.

The sampling error was also evaluated because the sampling interval influenced the calculation of statistics such as the population mean and variance. With a sample size of 2000 per measurement point, the uncertainty was evaluated as described by Benedict and Gould (1996) and the integral timescale $\tau_{i}$ in this method was calculated as presented by Nobach (2000). At a $95 \%$ confidence limit, we estimated that $50 \%$ of the velocity measurement points had an error of less than $1 \%$ and the remainder had an error of less than $8 \%$.

\section{RESULTS AND DISCUSSION}

The distances and velocities we recorded were nondimensionalised using the half-width of the diffuser, $d$, and the freestream velocity, $U_{\infty}$, respectively. The nearwake data are presented to highlight the flow physics of the diffuser and the aerodynamic effects of the geometrical alterations. As shown in Figure 8, the nearwake measurement planes (' $\mathrm{A}$ ' at $z / d=0.490$ and ' $\mathrm{C}$ ' at $z / d=-0.490)$ on either side of the centre plane 'B' $(z / d=$ 0 ) are positioned in line with the approximate cores of the longitudinal vortices.

3.1 Maximum-Force and Force-Reduction Regimes The maximum downforce and drag of the diffuser bluff body models were measured at the non-dimensionalised ride height of $h / d=0.191$. The maximum-force flow 
regime described by Senior (2002) comprises two longitudinal counter-rotating vortices at each side of the diffuser. Furthermore, the vortices were found to be steady and strong at the early part of the diffuser but became unsteady and more diffuse towards the exit. The axial speed at the core of the vortices at the exit was less than that of the surrounding flow due to vortex diffusion (Senior, 2002).

\section{REAR VIEW}

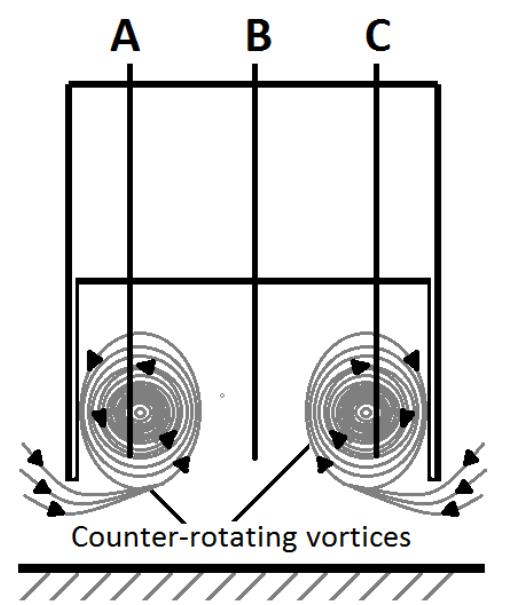

Figure 8 . The alignment of the measurement planes with the approximate locations of the diffuser vortices.

Figure 9 shows the baseline diffuser near-wake mean velocity measurements at $h / d=0.191$ for the two planes (' $A$ ' and ' $C$ ') at either side of the bluff body centreline. The position of the two planes enabled us to capture the near-wake velocity magnitude at the approximate positions of the vortex cores. In Figure 9(i) and (ii), the similarities in near-wake velocity magnitude profiles indicate that the diffuser flow is reasonably symmetric. Also, low velocities in the triangular region within the upper part of the near-wake planes infer the presence of the anticipated base flow recirculation. These low velocities are induced by the separated flow region near the base surface of the bluff body. Low velocities also dominate within the lower part of the near-wake planes. This implies that the cores of the vortex pair are diffused at the diffuser exit as reported by Senior (2002) and that the diffused vortices shown in Figure 9 have induced low velocities with increased turbulence in this region, as shown in Figure 10. Low turbulence was present on both planes in the immediate wake region (labelled 'Q') of the bluff body back plate, which indicated that the wake vortices are steady at that region (Figure 10). In addition, the higher-velocity region (labelled 'P') above the lowvelocity region at the lower part of the wake planes induced by the diffused vortices indicated that, towards the diffuser exit, the vortices have separated from the diffuser ramp (Figure 9).

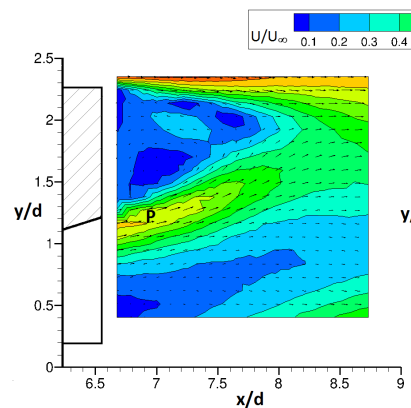

(i)

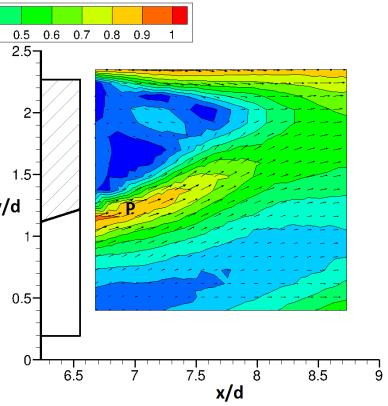

(ii)
Figure 9. Normalized mean $U$ contours for the plane diffuser at $h / d=0.191$ on (i) plane 'A' and (ii) plane 'C'.

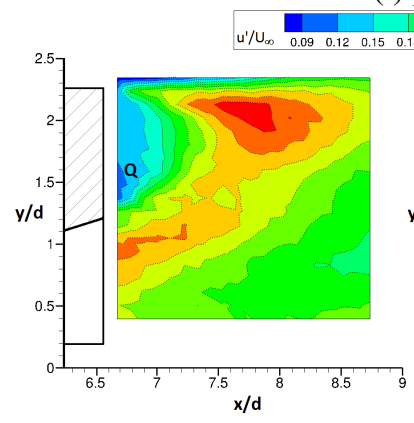

(i)

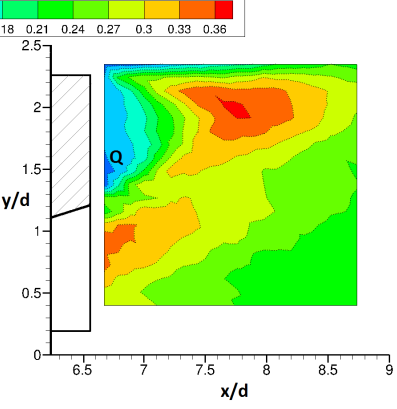

(ii)
Figure 10. Turbulence intensity $\left(u^{\prime} / U_{\infty}\right)$ contours for the plane diffuser at $h / d=0.191$ on (i) plane 'A' and (ii) plane 'C'.

Velocity measurements were taken at $h / d=0.153$ in the downforce-reduction regime. These reveal different velocity contour plots on planes ' $A$ ' and ' $C$ ', indicating that the diffuser mean flow is substantially asymmetric, as shown in Figure 11(i) and (ii). The asymmetric flow can be attributed to the breakdown of one of the longitudinal vortices as shown for plane ' $A$ ' in Figure 11(i). As reported by Senior (2002), the loss of downforce coincided with the development of flow asymmetry and the asymmetric 3-d boundary layer at the diffuser inlet induced a breakdown of one of the vortices.

The breakdown of the vortex shown for plane ' $A$ ' in Figure 11(i) allowed the low velocities of the upper separated flow region to dominate a large area of the diffuser near-wake. As a result, separated flow is prevalent over a substantial area of the near wake in plane ' $A$ ' and flow turbulence is enhanced further downstream of the wake as shown in Figure 12(i). However, higher velocities (close to freestream velocity) are prevalent near the diffuser exit for plane ' $\mathrm{C}$ ' in Figure 11(ii) even though the separated flow region close to the base surface of the bluff body is still present. The turbulence contours near the diffuser exit of plane ' $\mathrm{C}$ ' in Figure 12(ii) indicate a significant reduction in the areas of diffuse and high turbulence in the vortex close to the diffuser exit, as observed at $h / d=0.191$ in Figure 10(ii). Senior (2002) 
observed that the surviving vortex had separated from the diffuser ramp surface upstream of the diffuser exit and that the proximate area of the bluff body base plate wake was dominated by low turbulence on both planes.

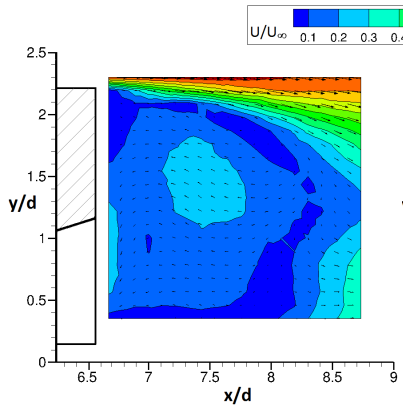

(i)

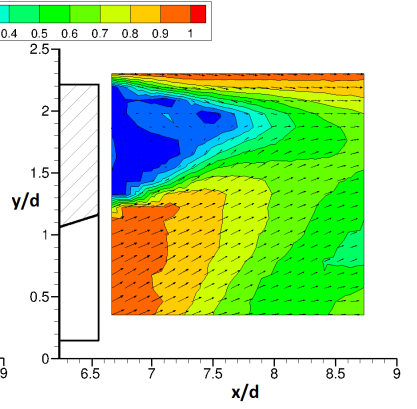

(ii)
Fig 10. Normalized mean $U$ contours for the plane diffuser at $h / d=0.153$ on (i) plane 'A' and (ii) plane ' $\mathrm{C}$ '.

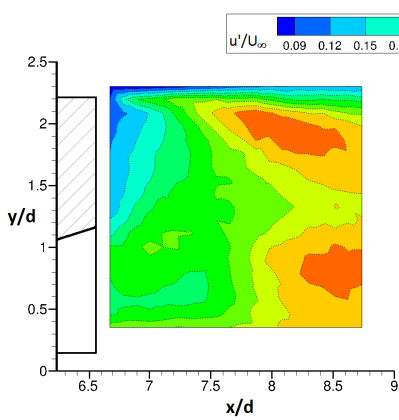

(i)

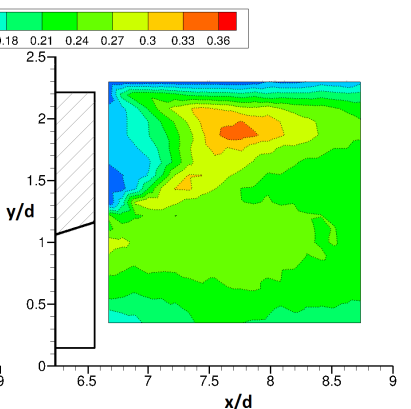

(ii)
Figure 12. Turbulence intensity $\left(u^{\prime} / U_{\infty}\right)$ contours for the plane diffuser at $h / d=0.153$ on (i) plane ' $\mathrm{A}$ ' and (ii) plane ' $\mathrm{C}$ '.

Notably, the higher velocities between the upper and lower low-velocity regions in Figure 9(ii) plane ' $\mathrm{C}$ ' reached a peak velocity approximately $11.1 \%$ higher than that of the corresponding measurement in the same area of plane 'A', as shown in Figure 9(i). The low velocities within the lower part of plane ' $\mathrm{C}$ ', which can be attributed to the diffused and turbulent part of the vortex at the diffuser exit, reached a velocity minimum that was up to $100 \%$ higher than the corresponding measurement in the same region of plane 'A'. Furthermore, the surviving vortex of the pair is on the side of the diffuser represented by the near-wake contours in plane ' $\mathrm{C}$ ' at a ride height of $h / d=0.153$ as shown in Figure 11(ii). These observations suggest that at $h / d=$ 0.191 , the vortex at plane ' $\mathrm{C}$ ' is stronger than that at plane ' $\mathrm{A}$ ', and for this reason the vortex at plane ' $\mathrm{C}$ ' in the asymmetric flow at a ride height of $h / d=0.153$ is still present.

\subsection{Near-Wake Streamwise Velocities}

The laser Doppler anemometry investigation of Zhang et al. (2004) was conducted on a similar diffuser body geometry (with the same length, height and width dimensions and the same diffuser angle) as the body used in the present study. However, unlike the current body, that used in the previous study had rounded lengthwise edges and a nose section formed by the merger of two radii $(741.5 \mathrm{~mm}$ and $131.6 \mathrm{~mm})$. Also, in the previous study, maximum downforce occurred at $h / d=0.217$ and the equivalent force-reduction ride height occurred at $h / d$ $=0.159$. Despite these differences, the streamwise velocities in the near-wake region of both diffuser bluff bodies reveal similarities in flow behaviour at a freestream and rolling-road velocity of $20 \mathrm{~ms}^{-1}$.

In Figure 13, the spanwise-plane results of Zhang et al. (2004) are compared (Figure 13(i)) with results from the streamwise planes (' $A$ ' and ' $C$ ') of the present study (Figures 13(ii) and 13(iii)). The streamwise velocity contours at $x / d=6.67$ show good flow symmetry for the maximum downforce ride height $(h / d=0.217)$ of the previous study. The near-wake flow behaviour also corresponds to the general flow symmetry at the streamwise near-wake planes ('A' and ' $C$ ') at the approximate vortex pair locations and maximum-force ride height $(h / d=0.191)$ of the present study. At $y / d=$ 1.1 , the normalised streamwise velocity contours for the previous and present studies reach highs of 1.05 and 0.85 respectively while at the approximate vortex cores $(y / d=$ $0.5)$, the velocity reaches a low of 0.15 for both studies.

The higher diffuser exit velocity at $y / d=1.1$ implies that the previous study has a higher diffuser inlet velocity.
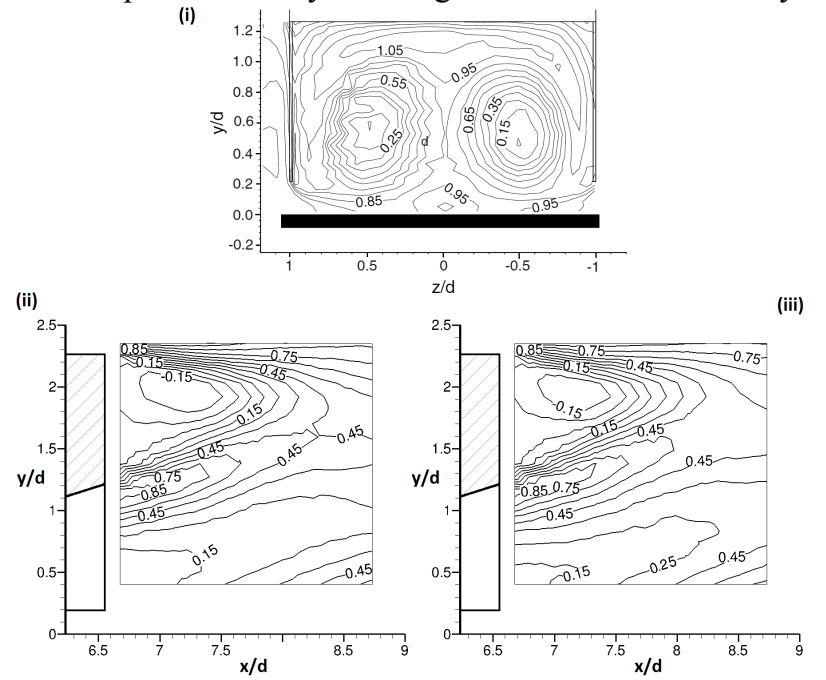

(iii)

Figure 13. Normalised mean $u$-component contours of plane diffuser near-wake at maximum-downforce ride height for (i) Zhang et al. (2004): spanwise plane at $x / d=$ 6.67 and ride height of $h / d=0.217$ (ii) present study: plane ' $\mathrm{A}$ ' at ride height of $h / d=0.191$ and (iii) present study: plane 'C' at ride height of $h / d=0.191$. 
This could be as a result of the rounded lengthwise edges of the flat underbody section enhancing diffuser inflow velocity with the curvature of the edges (hence creating a radial pressure gradient), or it could be due to the difference in shape of the nose section. Thus, maximum downforce generated by the previous study is $\sim 3 \%$ greater than that of the present study.

Flow asymmetry can be seen in Figure 14(i), where the near-wake streamwise velocity contours at $x / d=6.67$ and at the force-reduction ride height of $h / d=0.159$ for the previous study show the presence of a vortex on one side of the diffuser exit and a vortex breakdown on the other side. In the case of the present study at the equivalent force-reduction ride height of $h / d=0.153$, the near-wake asymmetric flow behaviour (Figures 14(ii) and 14(iii)) indicates similarities with the previous study. Vortex breakdown occurred at plane ' $A$ ', while on plane ' $C$ ' the vortex is present. On each side of the diffuser where the vortex is present for both studies, the normalised exit velocities reach a high of 0.85 at $y / d=$ 1.1 and on the other side where vortex breakdown and flow reversal occurs for both studies, the exit velocities reach a low of -0.15 at $y / d=0.6$. However, the sides of the diffuser where the vortex is present and vortex breakdown/flow reversal occurs appears to interchange between both studies. This suggests that the flow asymmetry of the force-reduction ride height may be bistable, although there was no evidence in our

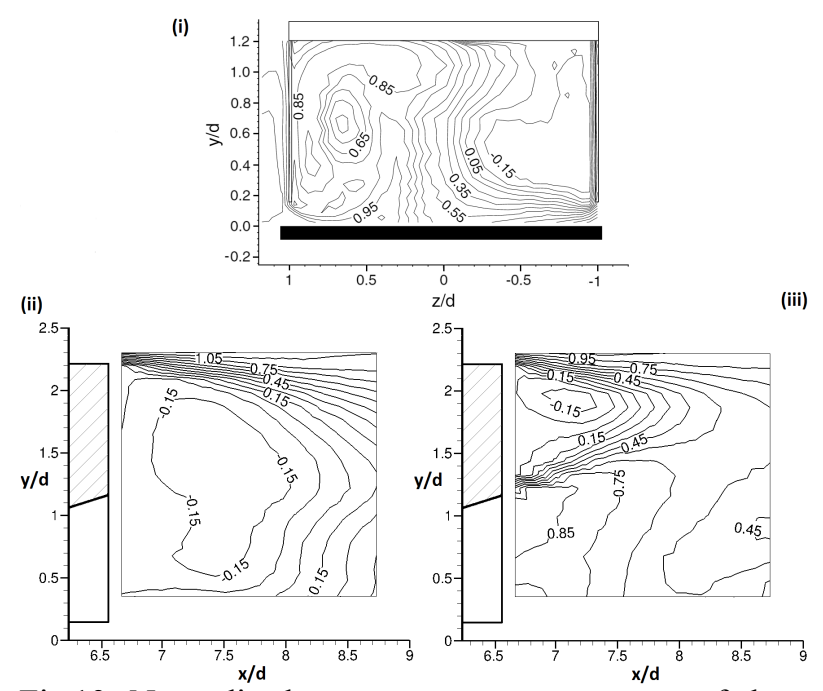

Fig 13: Normalised mean $u$-component contours of plane diffuser near-wake at downforce-reduction ride height for (i) Zhang et al. (2004): spanwise plane at $x / d=6.67$ and ride height of $h / d=0.159$ (ii) present study: plane 'A' at ride height of $h / d=0.153$ and (iii) present study: plane 'C' at ride height of $h / d=0.159$.

1 This refers to an upwash behind the diffuser body and is related to downforce generation (with clockwise or counter-clockwise directions defined by the freestream flow traveling from left to right). experiments of flow oscillation. The flow behaviour is a result of decreased boundary layer velocity with the reduction of ride height (therefore, reduced diffuser inlet area) beyond that of maximum downforce. It can then be surmised that the side of the diffuser where the boundary layer velocity is weaker determines which longitudinal vortex within the diffuser breaks down giving way to flow separation at the diffuser inlet (stated in Zhang et al. (2004)) and flow reversal.

\subsection{Influence of Diffuser Geometry}

The diffuser produces downforce through a velocitypressure relationship, such that fast-moving flow through the diffuser generates low static pressure within the diffuser. Accordingly, increasing the mass-flow in the diffuser at a given time will generate more downforce. Hence, flow control applications whether passive or active are beneficial only if they enhance the diffuser flow velocity.

The passive flow control measures we tested were principally geometric alterations: a convex bump and an inverted wing. The near-wake streamwise velocity $(u)$ and the change in velocity magnitude $(\Delta u)$ are presented to indicate the increase in downforce. The diffuser system we used does not have endplates sealed to the ground plane and also has a mass-flow inlet and exit. Therefore, the diffuser does not exist with closed boundaries. The geometric alterations caused a static pressure drop by increasing flow velocity, reducing separation and boundary layer thickness close to the diffuser exit. As a result, the new geometries increased the outflow from the diffuser. This reflects the interaction between the modified diffuser geometries and the circulation around the body, such that a greater net counter-clockwise ${ }^{1}$ circulation around the entire body implies an increase in downforce.

As shown in Figure 15, the boundaries (dashed lines) of the higher streamwise velocities in the near-wake of the modified diffuser bluff bodies (particularly at the contour regions indicated by ' $a$ ', ' $b$ ' and 'c') were shifted further downstream than the corresponding boundaries of the plane diffuser, indicating that circulation around the body had increased. Also, as indicated in Figure 15(ii) and (iii), the inverted wing appeared to enhance the streamwise velocity more than the convex bump. The deficit velocity magnitude contour region (labelled ' $S$ ') of the difference between $U$ of the plane diffuser and $U$ for each of the flow control methods as shown in Figure 16(i) and (ii) also indicated that the passive flow control methods enhanced the diffuser flow velocity and, thus, net counter-clockwise circulation around the bluff body. 


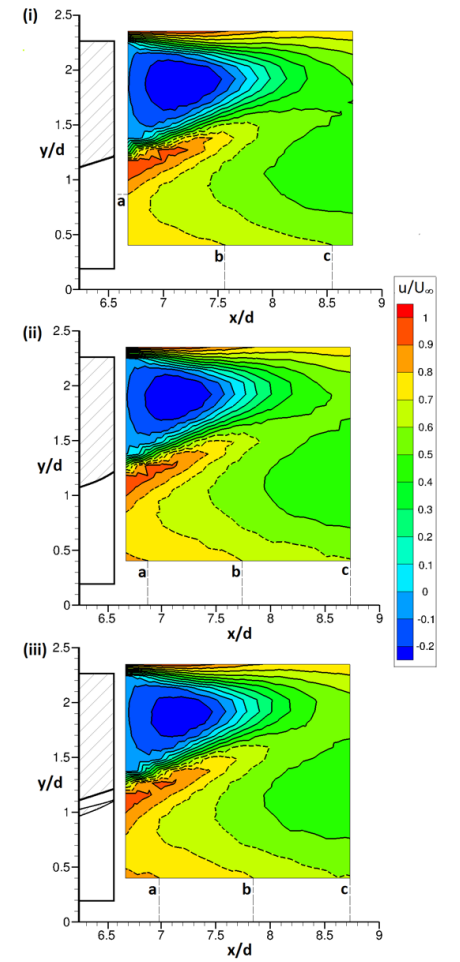

Figure 15. Plane ' $\mathrm{B}$ ' normalized mean $u$-component contours at $h / d=0.191$ for (i) plane diffuser, (ii) diffuser with a convex bump, and (iii) diffuser with an inverted wing.

\subsection{Near-Wake Flow Structures}

The near-wake of bluff bodies is dominated by separated flow. The separated flow of the near-wake region in turn dominates the aerodynamic drag of bluff bodies, the pressure drag component of which can be attributed to the pressure difference between the high pressure (stagnation point) on the front surface of the bluff body and the low pressure on the base surface induced by the separated flow at that region. The flow structures at planes ' $\mathrm{A}$ ' to ' $\mathrm{C}$ ' of the near-wake of the diffuser bluff body (Figures 17-19) indicated the presence of the separated and recirculating flow in this region. Also, the flow exiting the diffuser was found to shape the recirculating flow in the near-wake region of the bluff body.

These observations agree with earlier reports that the near-wake at the centreline of a bus-shaped bluff body was dominated by upper and lower counter-rotating vortices (Krajnovic and Davidson, 2001). In addition, the lower vortex was found to have originated from the underbody flow traveling through the ground clearance of the bluff body. Likewise, Puglisevich and Page (2011) discovered that for a bluff body with a diffuser, the lower vortex of the near-wake generated by the flow exiting the diffuser dominated the near-wake region, reducing the size of the upper vortex generated by the separated flow over the end of the bluff body top surface.

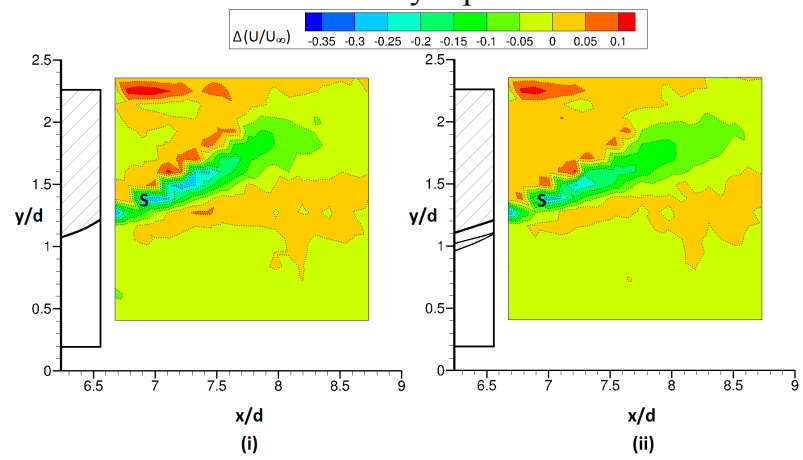

Figure 16. Plane 'B' normalized mean $\Delta U$ contours at $h / d$ $=0.191$ between plane diffuser and (i) diffuser with a convex bump, and (ii) diffuser with an inverted wing.

Figure 17 shows the flow structure at the maximum downforce ride height of $h / d=0.191$, suggesting that the lower vortex induced by the upward diffuser exit flow dominated the near-wake region of the baseline diffuser. This lower vortex is larger in size and appeared to have reduced the size of the upper vortex. The recirculating flows of the upper and lower vortices meet at a saddle point at a height of approximately $y / d=2.0$ and a streamwise distance of approximately $x / d=7.65$. Figure 17 also reveals flow angularity differences between planes ' $\mathrm{A}$ ' and ' $\mathrm{C}$ ' even though the corresponding velocities in Figure 9 reveal good flow symmetry. In contrast, a different near-wake flow structure appeared on plane 'A' at $h / d=0.153$ confirming the asymmetry of the diffuser flow in the force reduction flow regime (Figure 18).

On plane 'A' in Figure 18, the lower vortex induced by the flow exiting the diffuser flow is non-existent, indicating that the streamwise vortex on that side of the diffuser is also non-existent. The upper near-wake vortex is shown to dominate the wake of the bluff body. Furthermore, the upper vortex dominance appears to transform into a reverse flow which travels into the diffuser exit to replace the non-existing streamwise flow.

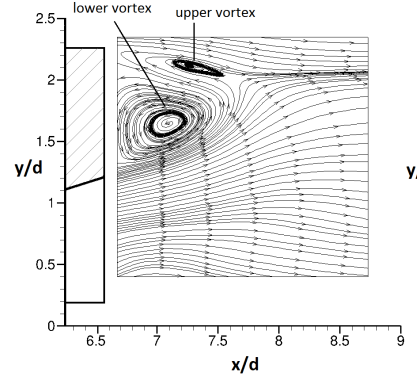

(i)

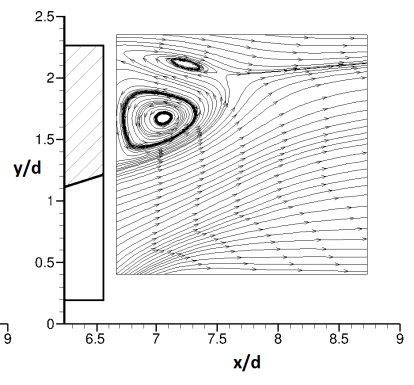

(ii)
Figure 17. Mean $U$ flow structures for the plane diffuser at $h / d=0.191$ on (i) plane 'A' and (ii) plane 'C'. 


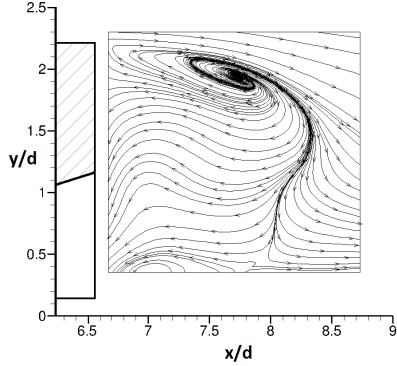

(i)

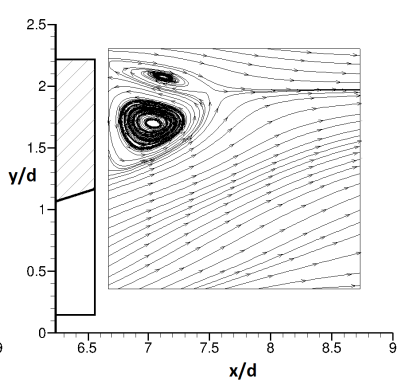

(ii)
Figure 18. Mean $U$ flow structures for the plane diffuser at $h / d=0.153$ on (i) plane ' $\mathrm{A}$ ' and (ii) plane ' $\mathrm{C}$ '.

The saddle point, where the upper and lower vortices meet, is no longer visible and has been replaced by a flow boundary that separates the reversed flow of the upper vortex and the streamwise wake downstream of the reversed flow. The flow boundary stretches downwardsfrom the upper vortex by a significant fraction of the bluff body height. The flow structure in plane ' $C$ ', however, did not change by comparison with the higher ride height, with the lower vortex remaining dominant over the upper vortex.

The centreline (plane ' $\mathrm{B}$ ') wake flow structures for the baseline and modified diffusers are compared in Figure 19, revealing that at the maximum downforce ride height $(h / d=0.191)$ the wake flow structure of the baseline diffuser is similar to that of diffusers with passive flow controls. The upper and lower counterrotating vortices remain present, with the lower vortex dominant. However, the lower vortex at the centreline plane for all three diffusers (Figure 19) is smaller than the lower vortex at planes ' $A$ ' and ' $C$ ' for the same ride height (Figure 17). This implies that the exit flow of the longitudinal streamwise vortices along both lengthwise sides of the diffuser is responsible for the increase in size of the lower vortex in the near-wake region.

Figures 20 and 21 highlight the differences in flow physics at planes ' $A$ ' and ' $B$ ' between the plane and modified diffusers in terms of velocity magnitude and turbulence intensity. The negative contours in Figure 20 indicate the plane diffuser's deficit in velocity magnitude compared to the modified diffusers. However, contour size differences in areas of positive velocity magnitude differences show higher velocity regions above and distinctly below the base plate at plane ' $A$ ' relative to plane ' $\mathrm{B}$ '. This explains the larger size of the lower vortex compared to the upper vortex in plane ' $A$ ' when comparing Figures 17 and 19. In addition, the contour regions of relative increase in turbulence intensity levels in Figure 21 indicate an increase in flow turbulence on planes ' $\mathrm{A}$ ' and ' $\mathrm{B}$ ' in the modified diffusers compared to the plane diffuser. Contour regions of relative decrease in turbulence indicate a turbulence deficit in the plane diffuser compared to the modified diffusers. Notably, increased areas of turbulence deficit appear in the lower regions of plane ' $A$ ' relative to plane ' $B$ '. This further confirms the presence of the turbulence generated by the diffused part of the longitudinal vortices at the diffuser exit.

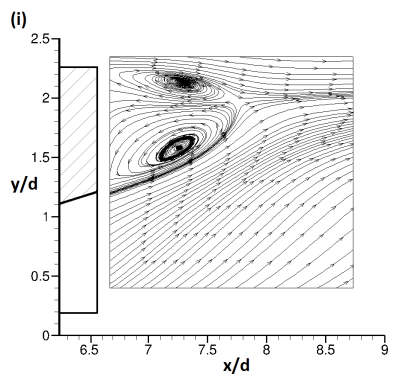

(ii)

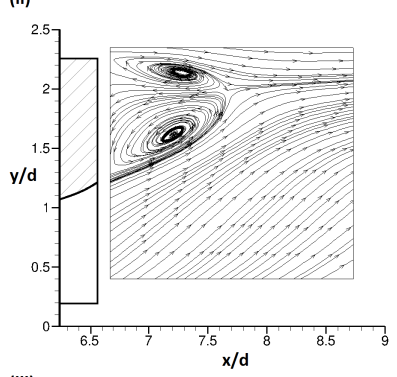

(iii)

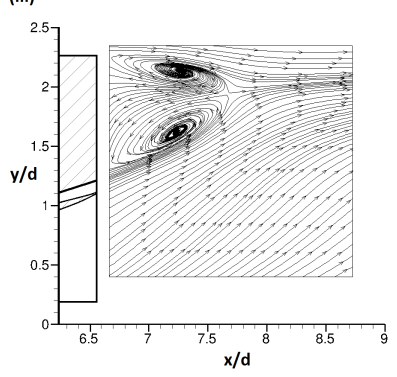

Figure 19. Plane ' $\mathrm{B}$ ' mean $U$ flow structures at $h / d=$ 0.191 for (i) plane diffuser, (ii) diffuser with a convex bump, and (iii) diffuser with an inverted wing.

Furthermore, the addition of the passive control devices was accompanied by an increase in drag. At the maximum downforce and drag ride height of $h / d=0.191$, the coefficient of drag increased from that of the plane diffuser by $1.8 \%$ (with the convex bump) and $1.8 \%$ (with the wing). For a 2-d incompressible flow (with density $\rho$ ) traveling around a body, the profile drag, $D$, (expressed in Equation 1) can be quantified by the difference in upstream velocity $U_{\infty}$ and downstream flow velocity $U_{w}$ at the near-wake area plane $\mathrm{d} A$ (Barlow et al., 1999).

$D=\iint \rho U_{w}\left(U_{\infty}-U_{w}\right) \mathrm{d} A$

This means that at the wake of the diffuser bluff body, there is a loss in flow momentum relative to upstream flow and an increase in drag corresponds to an increase in loss of flow momentum. Therefore, the 
relative velocity magnitude differences $(\Delta U)$ and the corresponding turbulence differences $\left(\Delta u^{\prime}\right)$ across the near-wake planes (Figures 20 and 21) are in general terms related to the additional profile drag induced by the convex bump and the inverted wing.
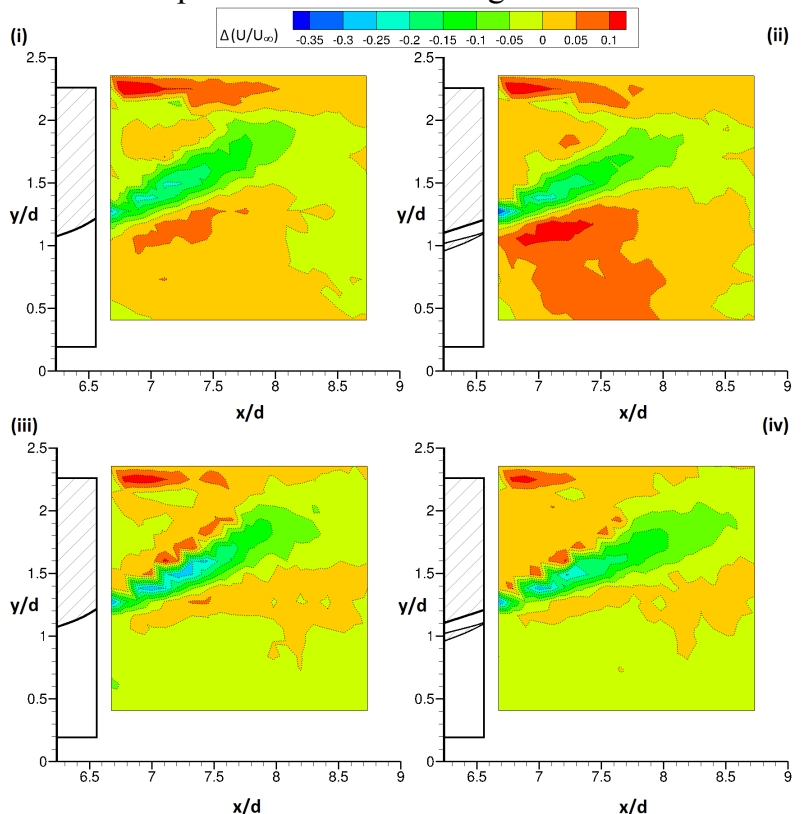

Figure 20. Normalized mean $\Delta U$ contours at $h / d=0.191$ between plane diffuser and (i) diffuser with a convex bump: plane 'A', (ii) diffuser with an inverted wing: plane 'A', (iii) diffuser with a convex bump: plane 'B', and (iv) diffuser with an inverted wing: plane 'B'.
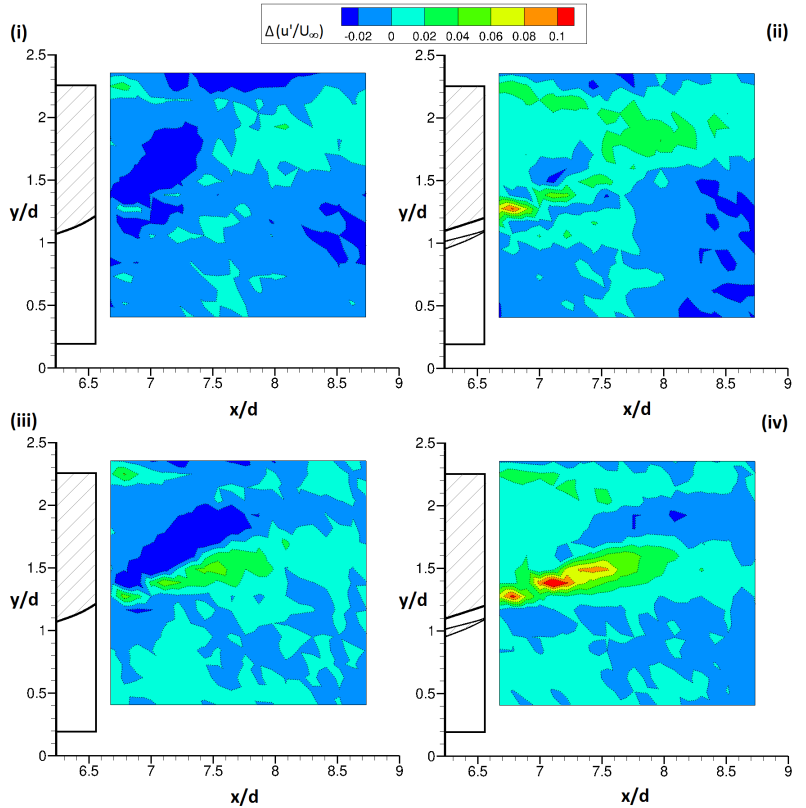

Figure 21. Normalized $\Delta u^{\prime}$ contours at $h / d=0.191$ between plane diffuser and (i) diffuser with a convex bump: plane 'A', (ii) diffuser with an inverted wing: plane 'A', (iii) diffuser with a convex bump: plane 'B', and (iv) diffuser with an inverted wing: plane ' $\mathrm{B}$ '.

\subsection{Near-Wake Circulation}

The flow physics of the circulation $\Gamma$ on the near-wake planes can provide additional information on the lift generated by a bluff body. The Kutta-Joukowski lift theorem relates the circulation around a 2-d body to the lift acting on the body. It also states that the circulation around a lift-generating body is finite and is related to the body's boundary layer vorticity. As a result, a positive lift force on the body is accompanied by a net circulation increase ("clockwise" circulation) associated with a "counter-clockwise" boundary layer vorticity at the trailing edge. The lift force, $L$, generated, or in this case the body downforce, is expressed as a product of the fluid density, freestream velocity and the circulation $(L=$ $\rho U_{\infty} \Gamma$ ). The circulation can be defined (Equation 2) as a line integral of the flow velocity vector $\vec{U}$ with respect to distance $\mathrm{d} l$ around a closed curve $S$, or by applying Stokes' theorem (Equation 3) where circulation is defined as the integral over an enclosed surface $A$ of the curl of the velocity field (vorticity) $\vec{\omega}$ for an area element $\overrightarrow{d A}$ in the normal direction $\hat{n}$ of the vector field across the surface.

$\Gamma=\oint_{\mathrm{S}} \vec{U} \cdot \mathrm{d} l$

If $\vec{\omega}=\nabla \times \vec{U}$, then

$\Gamma=\oint_{\mathrm{S}} \vec{U} \cdot \mathrm{d} l=\iint_{\mathrm{A}} \vec{\omega} \cdot \mathrm{d} A$

If we assume flow symmetry about the body centreline plane, examination of the relative changes in the wake circulation in this plane, if independent of other vortical flow structures such as in the $y-z$ plane, changes in the circulation in the $x-y$ plane should correspond to relative changes of lift and drag generated by the body. Therefore net wake circulation levels were calculated in plane ' $\mathrm{B}$ ' by expressing $\omega$ as the curl of the $u-v$ LDV velocity vector field and applying Stokes' theorem across the surface of plane 'B' (Figure 22).

Hence this result would infer, based on the centreline plane, a counter-clockwise circulation exists over the body, consistent with the generation of a net downforce. The result in part also corresponds to observed streamwise velocity differences between the plane diffuser and the modified diffusers illustrated in Figure 15 and discussed in Section 3.2 of this paper. Table 1 outlines the relative changes in circulation with respect to the baseline diffuser and compares these changes to lift and drag data for $h / d=0.191$.

It can be seen that the circulation integration predicts a reduction in lift in the centerline $x-y$ plane with respect to the baseline case of up to $23.5 \%$ for the diffuser bump. If this is compared to the change in overall body lift and drag coefficients, there is no correlation with either the magnitude or sense of the change, with respect to the 

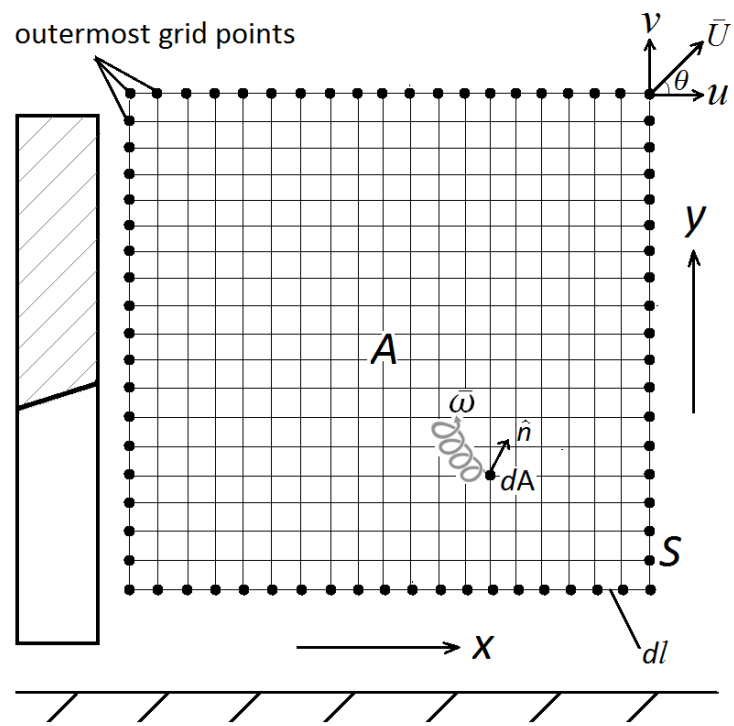

Figure 22. Schematic of the vector field across plane 'B'.

\begin{tabular}{lrrr}
\hline Diffuser Type & \multicolumn{1}{c}{$\boldsymbol{C}_{\boldsymbol{D}}$} & \multicolumn{1}{c}{$\boldsymbol{C}_{\boldsymbol{L}}$} & $\boldsymbol{\Gamma}\left(\mathbf{m}^{\mathbf{2}} \mathbf{s}^{\mathbf{1}} \mathbf{)}\right.$ \\
\hline Baseline diffuser & 0.4637 & -1.915 & -1.092 \\
\% from baseline & - & - & - \\
\hline Diffuser with bump & 0.4721 & -1.954 & -0.836 \\
\% from baseline & +1.8 & +2.0 & -23.5 \\
\hline Diffuser with wing & 0.4722 & -1.973 & -0.926 \\
\% from baseline & +1.8 & +3.0 & -15.3 \\
\hline
\end{tabular}

Table 1: Comparisons of relative changes in $x-y$ plane circulation to changes in body lift and drag.

baseline case. Therefore, although the diffuser geometry changes, including the bump and the wing, are clearly influencing the $x-y$ levels of circulation, the dominant flow features that determine the lift and drag characteristics on the body, reside in the $y-z$ plane. The dominant effect of these $y-z$ flow structures also is evidenced by minimal changes in overall lift and drag when the diffuser geometry is changed in both cases. Integration of circulation in the $y-z$ plane would be expected to support this inference through significantly higher levels of $y-z$ circulation, from flow structures reported by previous researchers (Jowsey and Passmore, 2010; Zhang et al., 2004) and evidenced in the LDV data presented in Section 3. Thus it must be concluded, if substantial changes in lift and drag are to be obtained, any diffuser geometry changes must influence the $y-z$ plane flow structures in preference to any $x-y$ plane flow structures. Further LDV measurements in the $y-z$ plane are required to validate this effect.

\section{CONCLUSION}

Our experimental investigation to ascertain the velocities and flow structures of the near-wake of a bluff body fitted with a ground-effect diffuser supports the following conclusions.

- The wake on either side of the bluff body centreline in the maximum-force flow regime consisted of low-velocity flow in the upper and lower parts of the wake and a higher-velocity flow between these regions.

- The wake at the side of the diffuser in the forcereduction flow regime (the site of vortex breakdown) largely consisted of a low-velocity flow, whereas on the other side, low-velocity flow dominated the upper wake region and higher velocities dominated the lower region.

- On the centreline plane of the wake in the maximumforce flow regime, streamwise velocity and velocity magnitude increased downstream of the diffuser exit with the alteration of the diffuser geometry (passive flow control).

- Flow behaviour such as the reasonable flow symmetry (in the maximum-force flow regime) and asymmetry (in the force-reduction flow regime) on the streamwise $(x-y)$ planes of the present study is in agreement with the flow behaviour of the respective diffuser flow regimes on spanwise $(y-z)$ planes of previous studies.

- In general, the upwardly-inclined flow exiting the diffuser induced a counter-clockwise vortex in the wake of the diffuser and this reduced the size of the upper clockwise vortex induced by the flow above the bluff body. However, in the force-reduction flow regime, the near-wake flow structure on the side of the diffuser featuring vortex breakdown partly consisted of an upper recirculating flow and a reverse flow travelling into the diffuser exit.

- Investigation of the $x-y$ centreline plane levels of circulation indicated the diffuser bump to have the most significant effect on the $x-y$ wake circulation. However, the relative changes in this circulation plane, with respect to the baseline case, were an order of magnitude greater than the overall changes in body lift and drag. Therefore it must be concluded that the $y-z$ plane circulation dominates the body lift and drag characteristics and so future studies of diffuser geometry should focus on influencing the circulation in this plane in preference to the $x-y$ plane.

ACKNOWLEDGEMENT- The first author gratefully acknowledges financial support from the Niger Delta Development Commission (NDDC) of Nigeria and from 
Cranfield University.

\section{REFERENCES}

Barlow, J. B., Rae, W. H. and Pope, A. (1999). Lowspeed wind tunnel testing. $3^{\text {rd }}$ edn. Wiley, New York, pp. 176.

Benedict, L. and Gould, R. (1996). Towards better uncertainty estimates for turbulence statistics. Exp. Therm. Fluid Sci. 22, 2, 129-136.

Buchave, P., George Jr, W. K., Lumley, J. L. (1979). The measurement of turbulence with the laser doppler anemometer. Annu. Rev. Fluid Mech. 11, 443-503.

Cooper, K. R., Bertenyi, T., Dutil, G., Syms, J., and Sovran, G. (1998). The aerodynamic performance of automotive underbody diffusers. SAE Technical Report. Paper 98-0030.

Cooper, K. R., Sovran, G. and Syms, J. (2000). Selecting automotive diffusers to maximise underbody downforce. SAE Technical Report Paper 2000-010354.

Dring, R. P. (1982). Sizing criteria for laser anemometry particles. J. Fluid Eng-T ASME 104,15-17.

Ehirim, O. H. (2017). Aerodynamics and performance enhancement of a ground-effect diffuser. PhD Thesis, Cranfield University, UK.

George, A. R. (1981). Aerodynamic effects of shape, camber, pitch on ground-vehicle bodies. J. Fluid EngT ASME 103, 4, 631-637.

George, A. R. and Donis, J. (1983). Flow patterns, pressures and forces on the underside of idealised ground effect vehicles. Proceedings of the ASME Fluids Engineering Division Symposium on Aerodynamics of Transportation II, 7, 69-79.

Hucho, W-H. (1998). Aerodynamics of road vehicles. 4th Revised. (ed), Society of Automotive Engineers, Washington, DC, USA, Chap. 8, pp. 392-395.

Jowsey, L. and Passmore, M. (2010). Experimental study of multiple-channel automotive underbody diffusers. $P$. I. Mech. Eng. D-J. Aut., 224, 7, 865-879.

Jowsey, L. (2013) An experimental study of automotive underbody diffusers. PhD thesis, Loughborough University, UK.

Katz, J. (2006). New directions in race car aerodynamics. 2nd Revised (ed) Bentley Publishers, Cambridge, Massachusetts, Chap. 6. pp. 221-222.

Knowles, K. and Finnis, M. V. (1998). Development of a new open-jet wind tunnel and rolling road facility. In: 2nd MIRA International Conference on Vehicle Aerodynamics, Coventry, UK.

Knowles, R. (2005). Monoposto racecar wheel aerodynamics: investigation of near-wake structure and support-sting interference. $P h D$ thesis, Cranfield University, UK.

Krajnovic, S. and Davidson, L. (2001). Large-eddy simulation of the flow around a ground vehicle body. SAE World Congress, Paper 2001-01-0702.

McKeon, B. et al. (2007). Velocity, vorticity and Mach number. In Tropea C., Yarin A., Foss J. F. (eds.) Springer Handbook of Experimental Fluid Mechanics, Springer-Verlag Berlin Heidelberg, Chap. 5, pp. $296-$ 309.

Nobach, H. (2000). A global concept of autocorrelation and power spectral density estimation from LDA data sets. In: 10th International Symposium on Applications of Laser Techniques to Fluid Mechanics, Instituto Superior Técnico, Lisbon, Portugal.

Puglisevich, L. S. (2013). Large eddy simulation for automotive vortical flows in ground effect. PhD thesis, Loughborough University, UK.

Puglisevich, L. S. and Page, G. (2011). Large eddy simulation of the flow around a diffuser-equipped bluff body in ground effect. Proceedings of $A S M E$ International Mechanical Engineering Congress \& Exposition, pp. 313-322.

Senior, A. (2002). The aerodynamics of a diffuserequipped bluff body in ground effect. $P h D$ thesis, University of Southampton, UK.

Senior, A. E. and Zhang, X. (2001). The force and pressure of a diffuser-equipped bluff body in ground effect. J. Fluid Eng-T ASME 123, 1, 105-111.

Sovran, G. (1994). The kinematic and fluid-mechanic boundary conditions in underbody flow simulation. Proceedings of the CNR-Pininfarina Workshop on Wind Tunnel Simulation of Ground Effect, Turin, Italy.

Toet, W. (2013). Aerodynamics and aerodynamic research in Formula 1. Aeronaut. J. 117, 1187, 1-25.

Zerihan, J. and Zhang, X. (2000). Aerodynamics of a single-element wing in ground effect. J. Aircraft 37, 6, 1058-1064.

Zhang, X., Senior, A. and Ruhrmann, A. (2004). Vortices behind a bluff body with an upswept aft section in ground effect. Int. J. Heat Fluid Fl. 25, 1-9.

Zhang, X., Toet, W., and Zerihan, J. (2006). Ground effect aerodynamics of race cars. J. Fluid Eng-T ASME 59, 1, 33-49. 